\title{
La Sanidad Militar Operacional, en el contexto multinacional
}

\author{
Chereguini Pavón E. ${ }^{1}$, Blasco Ferrándiz R.2
}

Sanid. mil. 2015; 71 (2): 109-116; ISSN: 1887-8571

\begin{abstract}
RESUMEN
El apoyo sanitario constituye una importantísima capacidad a activar en las operaciones militares convencionales, pero incluso más aún en las operaciones en curso y futuras. La aportación del apoyo sanitario suficiente a las operaciones militares es crítica y las deficiencias en las capacidades sanitarias desplegadas han constituido un fuerte factor limitante para las operaciones militares conjuntas multinacionales. Además de la ausencia de proyectos de licitación comunitarios, la disponibilidad de personal sanitario es también una preocupación multinacional que afecta en un alto grado a la factibilidad del despliegue y al sostenimiento de las Formaciones Sanitarias de Tratamiento (FST), de capacidades tipo ROLE 2/3 durante periodos prolongados. Estas son las razones por las que las Organizaciones Internacionales (UE\&OTAN) están trabajando, en la actualidad, en los proyectos de "Pooling and Sharing", con el fin de normalizar las capacidades sanitarias nacionales y alcanzar un alto nivel de inter-operatividad para que los diferentes medios sanitarios funcionales (instalaciones, equipos, personal sanitario, procedimientos...) puedan ser integrados en la estructura marco de una Formación sanitaria de tratamiento multinacional (FSTM).
\end{abstract}

\section{Multinational military medical support}

SUMMARY: Medical support is a significant capability to activate in conventional military operations, but even more in current and future operations. The provision of sufficient medical support to military operations is critical and deficiencies in deployable medical capabilities have been a force limiter for multinational military joint operations. In addition to lack of common procurements projects, availability of medical personnel is also a common concern that severely affects the feasibility of deploying, and sustaining Medical Treatment Facilities (MTF), ROLE 2/3 capable for prolonged periods. These are the reasons why International Organizations (UE\&NATO) are currently working on projects of Pooling and Sharing in order to standardizing national medical capabilities and to achieve a high level of multinational interoperability so that different functional medical means (structures, equipments, personnel, procedures...) can be integrated within the framework structure of a Multinational Medical Treatment Facility (MMTF).

\section{INTRODUCCIÓN}

El apoyo sanitario constituye una capacidad fundamental en las operaciones militares de cualquier índole.

A pesar de que el tipo de guerra, de las contempladas en los criterios de la Alianza Atlántica en su artículo 5 (convencionales), en la que se producen gran cantidad de bajas en una amplia área geográfica, siguen constituyendo una preocupación, en la actualidad este escenario posiblemente se contempla como menos probable, siendo las de carácter localizado las que se llevan a cabo por los diferentes ejércitos encuadrados en la Unión Europea (UE) y en la Alianza Atlántica (OTAN).

La provisión del apoyo sanitario a las diferentes operaciones es una necesidad fundamental para el desarrollo de las mismas, dado que cualquier deficiencia constituye, sin duda, un factor limitante. La falta de proyectos conjuntos y la disponibilidad del personal sanitario, son algunos de los factores que pueden

${ }^{1}$ Cte. Veterinario.

${ }^{2}$ Cte. Farmaceútico.

AGRUHOC (BRISAN). Madrid. España.

Dirección para correspondencia: Enrique Chereguini Pavón. 913647151. echepav@ et.mde.es

Recibido: 20 de febrero de 2014

Aceptado: 10 de marzo de 2015 afectar a la viabilidad de un despliegue y del sostenimiento de las Formaciones Sanitarias de Tratamiento (FST,s). Es por ello, que Organizaciones Internacionales UE/OTAN están desarrollando en la actualidad proyectos como el denominado "Pooling and Sharing" (P\&S), que tienen por objeto normalizar las capacidades sanitarias de las diferentes naciones con la finalidad de alcanzar un alto grado de interoperabilidad multinacional, de modo que los diferentes recursos funcionales sanitarios (instalaciones, equipos materiales, personal, procedimientos, etc.) puedan ser integrados en la estructura marco de una Formación Sanitaria de Tratamiento, multinacional.

Con la finalidad de conseguir economías de escala, el apoyo sanitario en las diferentes operaciones, pretende ser realizado a través de una Formación Sanitaria de Tratamiento Multinacional (FSTM) constituida y regida por acuerdos y procedimientos conjuntos, implicando con ello la realización de un esfuerzo coordinado, entre los diversos países participantes, que evite/ reduzca al máximo las redundancias y duplicidades entre los proyectos que se están desarrollando en el seno de la UE y la OTAN, dado que todos ellos tienen como objetivo general la utilización eficiente y eficaz de los recursos, así como la interoperabilidad entre dichas Organizaciones Internacionales (OI,s) para alcanzar el mayor grado de preparación y cualificación en las FSTM,s, anteriormente comentadas.

La multinacionalidad en el apoyo sanitario, en futuras operaciones a desarrollar en el seno de la UE y/u OTAN, deben ser 


\section{E. Chereguini Pavón y R.Blasco Ferrándiz}

contempladas como un elemento para el desarrollo de conceptos, procedimientos y actuaciones, conjuntas y coordinadas, para conseguir una adecuada atención a las bajas.

\section{ALIANZA ATLÁNTICA}

En la reunión de la OTAN, celebrada en Lisboa en al año 2010, se puso de manifiesto la necesidad de reforzar la aptitud de la organización para alcanzar sus prioridades, satisfacer las necesidades de las operaciones en curso, hacer frente a los desafíos actuales y emergentes, siendo necesaria para ello la adopción de las llamadas "capacidades claves".

Posteriormente, en la cumbre celebrada en al año 2012 en Chicago, la OTAN aprueba la Iniciativa "Smart Defence" como una nueva forma de entender la generación de capacidades en materia de Defensa para el año 2020. Entre las que se establecen y desarrollan diferentes capacidades, que se plasman en unos 170 proyectos, teniendo todos ellos como objetivo "suprimir duplicidades, aumentar y fortalecer la cooperación, con la finalidad de realizar un reparto de responsabilidades y cargas económicas de los países aliados" (Fig 1 y 2). llos, en el codificado como Tier 1.15, se incorpora el Proyecto de "Pooling and Sharing of Multinational Medical Treatment Facilities" (MMTF), abierto a las naciones miembro y/o asociadas de la Alianza Atlántica.

El proyecto incorporado en el Tier 1.15, de apoyo sanitario a las operaciones militares multinacionales, está dirigido a constituir FSTM,s, especializadas para atender y cubrir todas las necesidades sanitarias que requiera cada tipo de misión, contándose para ello con los recursos humanos y materiales sanitarios militares que, los países miembros y/o asociados, ofrezcan y/o pongan a disposición de esta Iniciativa, de conformidad con los memorándum de entendimiento y los acuerdos de participación que cada gobierno establezca con la Alianza.

España, viene asistiendo y participando con representantes, pertenecientes al Cuerpo Militar de Sanidad, en las reuniones y trabajos celebrados en el "Military Medical Structures, Operations and Procedures" (MMSOP), Grupo de Trabajo del Committee of the Chiefs of Military Medical Services in NATO (COMEDS), de la iniciativa Smart Defence (OTAN), con el objetivo de estudiar, diseñar y/o definir estructuras, competencias y capacidades sanitarias, en la Función Logística de Sanidad, a prestar en las diferentes misiones multinacionales.

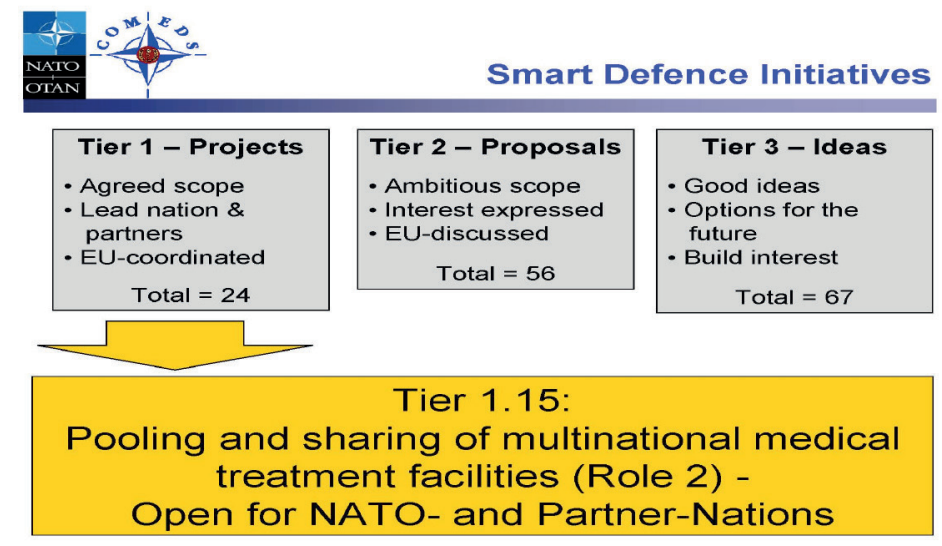

Figura 1. Iniciativas del Smart Defence OTAN.

\section{UNIÓN EUROPEA}

Desde la firma del tratado de Maastricht en 1992, la UE ha tratado también de realizar una estrategia propia en varias áreas, como por ejemplo en política exterior y en seguridad.

El establecimiento de una complementariedad con la OTAN, en materia de defensa, se puso de manifiesto a partir del 2004 con la creación de la Agencia Europea de Defensa (European Defence Agency, EDA); organismo especializado de la UE que está iniciando una aproximación sistemática en el establecimiento de áreas de cooperación para el desarrollo de diferentes capacidades. Este organismo constituye, a su vez, una plataforma de gran utilidad para la identificación, estudio y evaluación de diferentes necesidades y capacidades, entrelas que figura la de la Sanidad Militar, en las diferentes operaciones multinacionales.

En el mes de septiembre del año 2010, en el seno de la EDA, se presentó la Iniciativa Gante, en la que los ministros de defensa de todos los países, incluida España, acuerdan y se comprometen a realizar acciones que conduzcan a la concentración y compartición de recursos y capacidades, con la finalidad de eliminar duplicidades y realizar una contención de costes.

En mayo de 2011 la EDA, a través de su Junta de Gobierno, ordena realizar, en estrecha colaboración con el Comité Militar de la Unión Europea (EUMC), un listado de Iniciativas para impulsar el concepto de "Pooling and Sharing (P\&S)", mediante la identificación de los diferentes requerimientos operativos comunes, tratando de fomentar determinadas áreas que presenten un importante potencial para el futuro desarrollo de esta Organización. 


\section{La Sanidad Militar Operacional, en el contexto multinacional}

La Iniciativa europea del "Pooling and Sharing" cubre, de forma integral, el desarrollo de diferentes capacidades que van desde la selección y armonización de los requerimientos operativos hasta su sostenimiento, teniendo como objetivo estimular la creación de diferentes grupos de trabajo entre los estados miembros para:

- Establecer necesidades operativas.

- Identificar prioridades comunes.

- Proporcionar información que oriente al sector industrial europeo de la Defensa.

- Explorar oportunidades de colaboración.

España en el año 2011, inicia su participación en la Iniciativa Pooling and Sharing, colaborando en diferentes áreas como son la adquisición, sostenimiento, adiestramiento y empleo de fuerzas, siendo de destacar su presencia activa en las siguientes Iniciativas: MASUR (Maritime Surveillance), M3U (Multinational Modular Medical Unit), ENT (European Naval Training), AAR (Air to Air Refuelling), PT (Pilots Training) (Fig 3).

Estas Formaciones se denominan, en la Iniciativa del P\&S de la UE, Unidades Sanitarias Modulares Multinacionales (M3U,s), a diferencia de las de OTAN (que solo contemplan capacidad máxima de $2^{\circ}$ Escalón Sanitario, tipo ROLE 2), incluyen, además la capacidad de $3^{\text {er }}$ Escalón (tipos ROLE 2 y 3 ).

Estas M3U,s se configurarán atendiendo a la capacidad sanitaria específica y necesaria que requiera cada misión a apoyar, en función de la naturaleza de la misma (ayuda, humanitaria, interposición de la fuerza, de reconstrucción, desastres naturales, etc.), concentrando en ellas todos los esfuerzos y recursos necesarios para atender y recuperar las bajas sanitarias lo más cerca posible del punto donde se produzcan, en el Teatro de Operaciones (TO).

\section{SANIDAD MILITAR ESPAÑOLA}

Es importante señalar que las Unidades Sanitarias Militares, como por ejemplo las que dispone el ET, cambian su designación en el momento en que son desplegadas en el TO, pasando a denominarse Formaciones Sanitarias de Tratamiento (FST,s) que se van posicionando en varios escalones, en función de su misión y capacidades sanitarias a prestar.

Estas FST,s, designadas, en la actualidad, con la palabra ROLE, (papel o capacidad a desempeñar), derivada de la doctrina sanitaria OTAN, se clasifican en Role 1, Role 2 o Role 3, en función del Escalón Sanitario en el que se localizan y de la capacidad sanitaria que pueden desarrollar.

De conformidad con el PD4-616 de "Sanidad en operaciones" del MADOC (ET), para dar una mayor coherencia a nuestro escalonamiento sanitario con la nueva doctrina sanitaria OTAN, se establece que el Ejército Español dispone de una serie de FST,s diseñadas para ser desplegadas, en el correspondiente Escalón Táctico-Sanitario del Teatro de Operaciones (TO), en función del tipo de capacidad sanitaria que puede desempeñar,

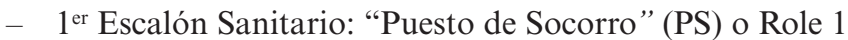
(OTAN) que despliega a vanguardia en el TO

- $2^{\circ}$ Escalón Sanitario Ligero: "Puesto de Cirugía Ligera Avanzada" (PCLA), que ofrece capacidad de Role 2 LM (Light Maneuver, OTAN) o bien de Role 1, en función de que disponga o no de equipo quirúrgico, respectivamente.

- $2^{\circ}$ Escalón Sanitario Reforzado: "Escalón Médico Avanzado Terrestre" (EMAT), equivalente a Role,s 2 LM o E. (Enhanced, OTAN) si se aumenta su capacidad de hospitalización y otros medios cuando proceda y el "Equipo de Cirugía Avanzada" (ECA), que dispone de dos equipos de cirugía. 2 ECA,s podrían constituir el núcleo de activación de un Role 2 LM.

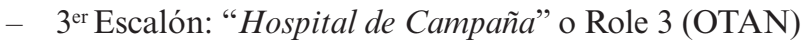

\section{EL HOSPITAL DE CAMPAÑA}

La FST más reciente de las FAS españolas es el Hospital de Campaña (HOC) del Ejército de Tierra (ET) que, encuadrado en la Agrupación de Hospital de Campaña (AGRUHOC) de la Brigada de Sanidad (BRISAN), dependiente de la Fuerza Logística Operativa (FLO), ofrece una capacidad sanitaria de tipo Role 3 (OTAN).

\begin{tabular}{|lll|}
\hline MASUR & Maritime Surveillance & $\begin{array}{l}\text { Desarrollar capacidades de inteligencia, vigilancia y reconocimiento, } \\
\text { así como la articulación de una red de vigilancia marítima para la } \\
\text { seguridad nacional y el despliegue en operaciones. }\end{array}$ \\
M3U & Multinational Modular Medical Unit & $\begin{array}{l}\text { Organizar Formaciones Sanitarias Multinacionales, constituidas } \\
\text { sobre la base de una nación marco, que cuente con contribuciones } \\
\text { complementarias de otros países. }\end{array}$ \\
EVT & European Naval Training & $\begin{array}{l}\text { Aumentar el adiestramiento e instrucción naval en ámbito de la } \\
\text { Unión Europea. }\end{array}$ \\
AAR & Air to Air Refuelling & $\begin{array}{l}\text { Fomentar y mejorar la capacidad de reabastecimiento en vuelo. } \\
\text { Obtención de economías de escala, mejorar la interoperabilidad y } \\
\text { solucionar problemas de transporte. }\end{array}$ \\
\hline
\end{tabular}

Figura 3. Iniciativas EDA. 


\section{E. Chereguini Pavón y R.Blasco Ferrándiz}

El origen del HOC surge en los llamados "Acuerdos de Praga," (OTAN 2002), en los que España se compromete a aportar a la Alianza Atlántica un Cuerpo de Ejército y sus diferentes apoyos logísticos, entre los que se encuentra el apoyo sanitario operacional, con un Hospital de Campaña de Tercer Escalón Sanitario.

Con esta adquisición España se convierte en el $8^{\circ}$ país del mundo que dispone de este tipo de FST.

En 2005 las FAS españolas inician las acciones para la adquisición del HOC que, tras la aprobación de su expediente de adquisición, en Consejo de Ministros de 23 de marzo de 2007, se resuelve con su adjudicación a una Unión Temporal de Empresas (UTE), el 24 de agosto de 2007.

En paralelo a las acciones mencionadas, el 23 de abril del 2007, en aplicación de la Instrucción Comunicada $\mathrm{n}^{\circ}$ 64, del Jefe de Estado Mayor del ET, se constituye la Agrupación de Hospital de Campaña (AGRUHOC), en la BRISAN de la Fuerza Logística Operativa (FLO) del ET, donde queda encuadrado orgánicamente el HOC (Fig. 4).

La entrega y recepción del material del HOC se desarrolló entre el mes de noviembre de 2008 y diciembre de 2010, dándose por finalizado el proceso de recepción y comprobación en el mes febrero de 2011.
- Verificar las capacidades disponibles así como los procedimientos necesarios y suficientes.

- Comprobar que el HOC y la AGRUHOC en su conjunto, está adiestrada en el conocimiento y aplicación de los procedimientos logísticos y/o sanitarios aprobados.

b. Específicos:

- Instrucción y adiestramiento del personal en los diferentes módulos no facultativos.

- Instrucción y adiestramiento en los módulos facultativos.

- Evaluación posterior por el equipo de evaluación del "NATO Centre of Excellence for Military Medicine" para, una vez superada, obtener la certificación del HOC como una FST de 3er Escalón de la OTAN (Fig. 6).

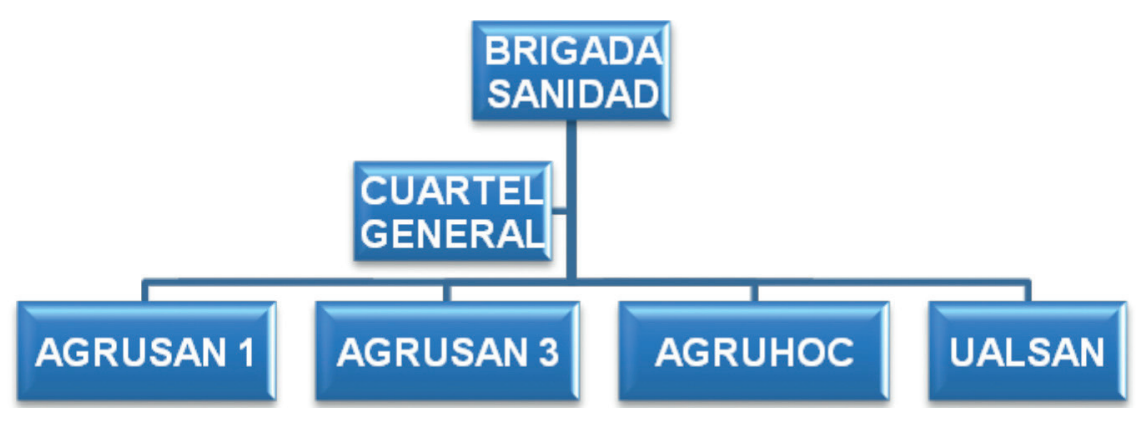

Figura 4. Organización de BRISAN.
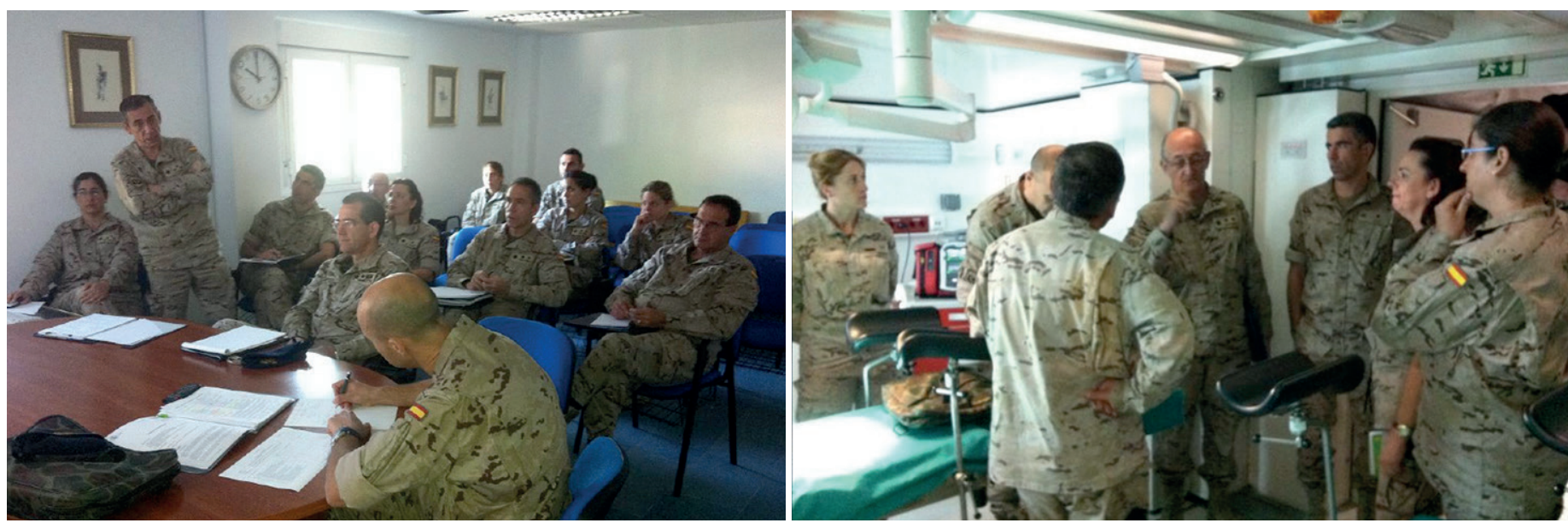

Figura 5. Evaluación FOC.

En mayo de 2011 se desarrolla el ejercicio de presentación que, junto con el adiestramiento e instrucción de su personal, hace posible que en mayo de 2012 se despliegue el HOC completo para ser evaluado de su "Capacidad Operativa Inicial" (Initial Operational Capability, (IOC)).

Un año más tarde, en mayo del 2013, la Agrupación Hospital de Campaña (AGRUHOC) supera la evaluación nacional de su Capacidad Operativa Final (Final Operational Capability, (FOC)), bajo el Ejercicio denominado "Batalla de Toro, 2013" (Fig. 5).

Dicha evaluación tuvo como objetivos:

a. Generales:

- Obtener la certificación nacional del HOC como Formación Sanitaria de Tratamiento de capacidad de Tercer Escalón Sanitario, tipo ROLE 3.

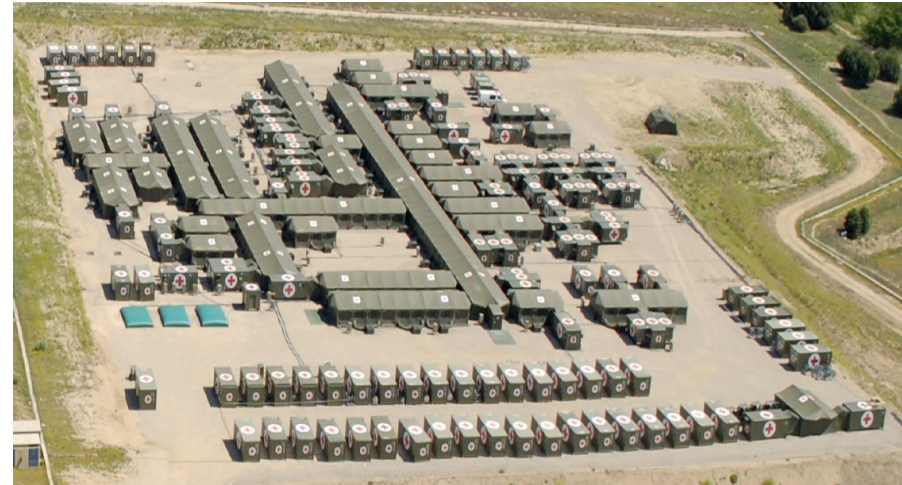

Figura 6. Vista general del Hospital de Campaña. 


\section{La Sanidad Militar Operacional, en el contexto multinacional}

El HOC del ET, ha sido la primera unidad sanitaria española evaluada, como FST con capacidad de Role 3, bajo estándares OTAN a través del Manual de Evaluación Sanitaria "AMedP-27", cuyo propósito es facilitar y proporcionar el marco de referencia necesario para que los países aliados certifiquen sus capacidades, módulos y/o FST,s, cuando se constituyan para apoyar una operación de la Alianza.

La certificación sanitaria en el campo militar, constituye el reconocimiento oficial de que bien el personal, módulos, protocolos, procedimientos, formaciones y/o componente de la fuerza ostentan la capacidad y/o requerimientos normalizados por los países miembros o bien, en caso negativo, determina e identifica los puntos críticos, riesgos potenciales y las acciones preventivas, correctoras o de mejora necesarias que deben ser implementadas.

La actual situación de la sanidad militar española, miembroparticipante en operaciones multinacionales, lideradas por OI,s, en las que se requieren FSTM,s, debería ser sometida a revisión para que la Función Logística de Sanidad Nacional se constituya en un único estándar para la Defensa, y estar en mayor consonancia con la logística y derroteros de la sanidad militar internacional.

Debemos también tener en cuenta los cometidos del planeamiento operacional, así como del adiestramiento e instrucción sanitaria de las FST,s, que competen a la Inspección General de Sanidad de la Defensa (IGESANDEF), como así lo dispone la Instrucción Técnica 2/2013 de 15 de Enero de la Subsecretaría de Defensa, por la que se asignan funciones y cometidos en el ámbito de la IGESANDEF y se describe su estructura orgánica (BOD $n^{\circ}$ 18/2013), como máximo órgano militar de las FAS encargado de facilitar y dotar de personal cualificado para cualquier FST que sea desplegada.

\section{MULTINACIONALIDAD DE LAS FST,S}

Las diferentes iniciativas en curso, anteriormente comentadas, relacionadas con el apoyo sanitario multinacional, van encaminadas a constituir FSTM,s a partir de aquellos medios materiales y personales que los diferentes países miembros y/o asociados de la OTAN/UE ponen a disposición en estos proyectos, teniendo en cuenta que son las "necesidades de atención sanitaria especificas e identificadas del T.O." las que determinarán en su proceso de planeamiento, la estructura y capacidades requeridas.

Como se ha citado con anterioridad, España está presente en los diversos foros internacionales, que mantienen entre sí una estrecha relación de colaboración y coordinación, con la finalidad de establecer sinergias y criterios comunes de actuación, ofreciendo FSTM,s de capacidad Role 2 (LM y E) al proyecto Smart Defence y de tipo Role 2 (LM y E), así como de Role 3 (HOC del ET) en el P\&S, suscribiendo, de este modo, su compromiso e interés de participación en la multinacionalidad de la sanidad militar, dado que esta representa una serie de ventajas como las que se citan a continuación:

- Cobertura de carencias de personal sanitario cualificado.

Unit).
- Intercambio de conocimientos y lecciones aprendidas de la experiencia y de diversos Centros especializados, como por ejemplo el Centre of Excellence for Military Medicine $(\mathrm{CoE})$ de la OTAN en Budapest.

- Normalizar Procedimientos conjuntos bajo los estándares de OTAN y de la UE.

- Fomentar el conocimiento del idioma inglés en el personal sanitario.

- Optimizar recursos materiales y personales.

- Racionalizar los costes.

- Utilizar recursos comunes para el desarrollo y sostenimiento de las operaciones multinacionales.

- Coordinación en la adquisición y mantenimiento de equipos.

- Etc.

La evolución en la Sanidad Militar operativa internacional, fruto de experiencias y de lecciones aprendidas, ha llevado a la UE a modificar conceptos y a redefinir unidades tradicionales como las MIMU,s de la OTAN (Multinationational integrated Medical Units) en otras denominadas M3U (Multinational Modular Medical Unit) incorporando el término "Modular" y eliminando el término "integrated," en cuanto este implica un nivel de compromiso demasiado exigente para los países participantes al incluir todas las capacidades sanitarias independientemente de la naturaleza de la misión (Fig. 7).

Figura 7. Evolución hacia el concepto M3U (Multinational Modular Medical

EU P\&S - «MEDICAL SUPPORT TO EU COMBINED MILITARY OPERATIONS»

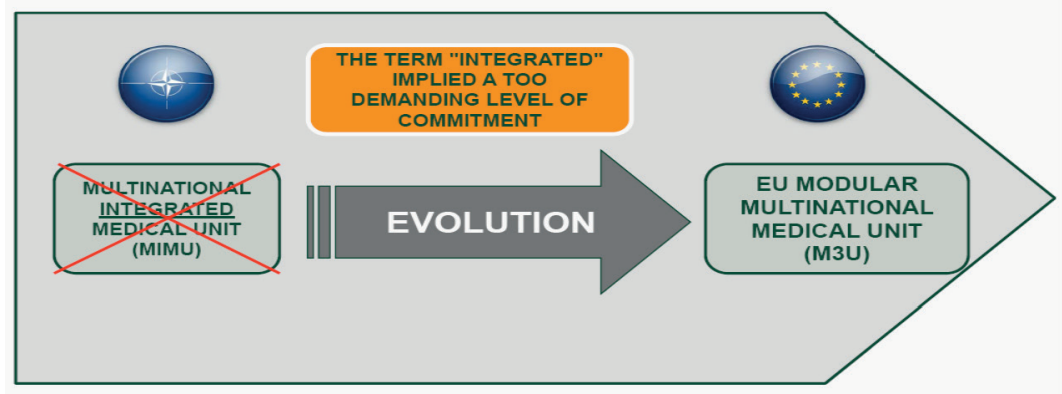

En la actualidad son dos los grupos de trabajo que están desarrollando los nuevos conceptos y doctrina sanitaria militar operativa: el MMSOP-WG (Military Medical Structures, Operation and Procedures Working Group en la Iniciativa Smart Defence de la OTAN), liderado en la actualidad por Francia e Italia y, el M3U-AHWG (Multinational Modular Medical Unit Ad Hoc Working Group), de la Iniciativa Pooling and Sharing de la EDA en la UE, liderado por Italia (Fig. 8).

Estos grupos de trabajo presentan como objetivo común la agrupación y/o configuración de instalaciones, recursos materiales y personales, así como procedimientos para conformar las diferentes FST,s, basadas en una "aproximación de tipo modular."

Así se distinguen:

$\checkmark$ Instalaciones de Tratamiento Sanitario Multinacional; Multinational Medical Treatment Facility (MMTF) de 


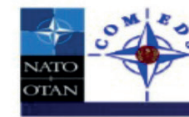

Projects in NATO/EU

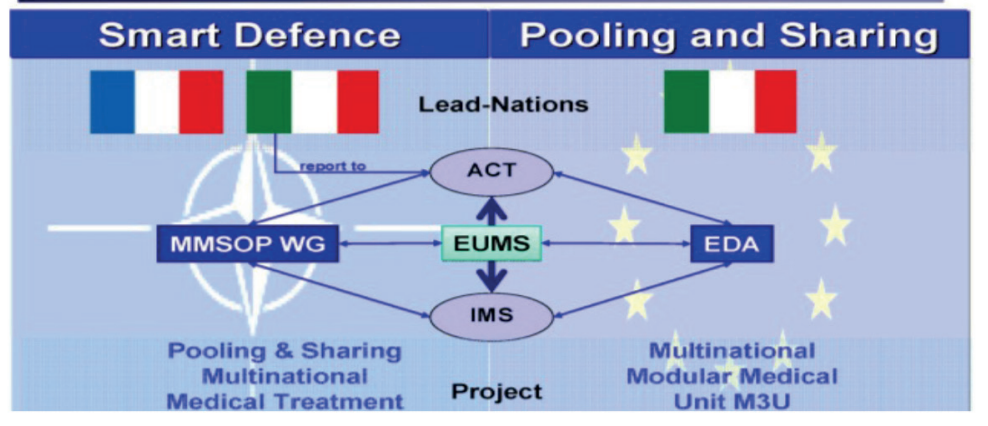

ACT: Allied Command Transformation IMS: Intelligent Manufacturing System

Figura 8. Relación Smart Defence y Pooling and Sharing. capacidad sanitaria tipo Role 2, en la iniciativa Smart Defence (OTAN).

$\checkmark \quad$ Unidades Sanitarias Modulares Multinacionales; Multinational Medical Modular Unit (M3U) en el proyecto de P\&S de la EDA (UE) para capacidades sanitarias de tipo Role,s 2 y 3.

El carácter modular de las FSTM,s es consecuencia del tipo de estructura y capacidades que la constituyen: Módulos sanitarios y Contribuciones complementarias (Fig. 9).

Módulo sanitario: implica una capacidad sanitaria completa e indivisible, que no se puede subdividir en otros sub-módulos, comprendiendo en sí mismo las instalaciones, su personal específico y/o cualificado, el equipamiento completo, los materiales, así como los procedimientos de actuación necesarios.

Contribuciones complementarias: elementos adicionales para ampliar las capacidades básicas modulares de la FST, en función de la naturaleza de la misión encomendada.

Según estos criterios, los módulos se configurarán en función del tipo, naturaleza y objetivos de la misión, representando el "Módulo Core" el núcleo o germen de una FSTM de capacidad de tipo Role 2, una capacidad funcional de carácter indivisible que cuenta con las siguientes capacidades sanitarias esenciales:

- Urgencias y clasificación.

- Cirugía inicial de control de daños.

- Diagnóstico.

- Hospitalización temporal de bajas.

- Mando y control.

El "módulo core" deberá estar diseñado y construido con las peculiaridades necesarias para actuar tanto en operaciones marítimas como terrestres, con mayor o menor capacidad de movilidad (Fig. 10).

En cambio, los Módulos Enhancing, "mejorantes", no son necesarios en su totalidad, por lo que solo deberán ser seleccionados aquellos verdaderamente necesarios, configurándose en

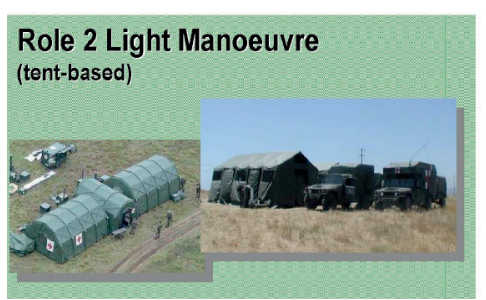

\section{Modified and tailored to the mission}

Role 2 with more static approach (tent/container-based or fixed buildings or naval platforms)

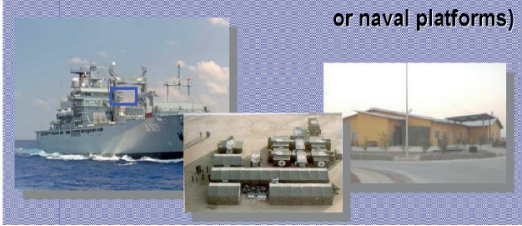

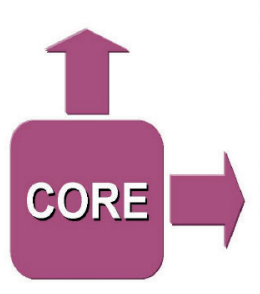

Figura 10. Tipo de FST en función del escenario operacional. especialización y/o dimensionamiento en función de las características y peculiaridades de la operación en que vayan a ser desplegados. En la actualidad su definición y composición es objeto de estudio en el seno de los grupos de trabajo MMSOP-WG y M3U-AHWG, con fines de estandarización y normalización siguiendo los criterios y directrices de OTAN y UE.

Una vez definida la composición y capacidades del considerado modelo básico de MMTF/M3U, éste deberá ser aprobado por los organismos directores de los proyectos Smart Defence (MMSOP) y de P\&S (EDA) para constituir un referente de partida en el planeamiento sanitario de una operación determinada.

De manera semejante, con la finalidad de dirigir, coordinar y configurar una determinada FST según la naturaleza y tipo de una operación, se establecen diferentes denomi- 
naciones para las naciones y tropas participantes, en función del papel que van a desempeñar.

Nación Líder (LN): país que dirige y coordina las actividades en los grupos de trabajo del MMSOP y de la EDA de cara a desarrollar la capacidad sanitaria de una MMTF/M3U básica, así por ejemplo Francia e Italia se han ofrecido como naciones líder para las iniciativas Smart Defence y P\&S respectivamente.

Nación Marco (FN): país o países que necesitan ser identificados para proporcionar y constituir el llamado módulo core, antes citado, con al menos:

- El Mando y Control, las Comunicaciones, Ordenadores y la Inteligencia (C4I).

- Servicios de Comunicaciones y de Información (CIS).

- Servicio de apoyo al combate. Combat Support Service (CSS).

- Protección a la Fuerza. Force Protection (FP).

- Normas y Procedimientos operativos. Standard Operation Procedures (SOP,s).

Estos países son los responsables de llevar a cabo el adiestramiento pre-despliegue, la validación y cualificación de todos los medios (humanos y materiales), así como hacer efectiva su integración en la Fuerza, en las actividades previas al despliegue de una M3U.

Nación Contribuyente con Tropa (TCN): estos países deben ser capaces de proveer módulos normalizados y/o contribuciones complementarias que cumplan con las normas y procedimientos acordados.

La M3U, resultante de recombinar los módulos (core y mejorantes) y las contribuciones complementarias, debe estar sometida a un proceso de validación por un organismo acreditado tanto para la Iniciativa Smart Defence (OTAN) como la de Pooling \& Sharing de la EDA (UE).

Los módulos y/o contribuciones aportados deberán ser validados por lo tanto por un Equipo de Evaluación Multinacional (MET), apoyado por el Centro de Excelencia para la Sanidad Militar de la OTAN (CoE) en Budapest (único organismo internacional acreditado para evaluaciones en el ámbito sanitario operacional por el momento). Este MET está constituido por personal evaluador cualificado y acreditado según lo establecido en el Medical Evaluation Manual (MEM), recogido en el AMedP-27 de la OTAN.

Por otra parte la validación de la FSTM (tipos Role 2/3), en la iniciativa P\&S, también será llevada a efecto por un Equipo de Evaluación Multinacional constituido al efecto, bajo la colaboración y dirección técnica del CoE de Budapest, en tanto se establezca un centro acreditado similar en la UE. Esta validación consistirá igualmente en evaluar la capacidad, eficiencia y eficacia de los elemen-
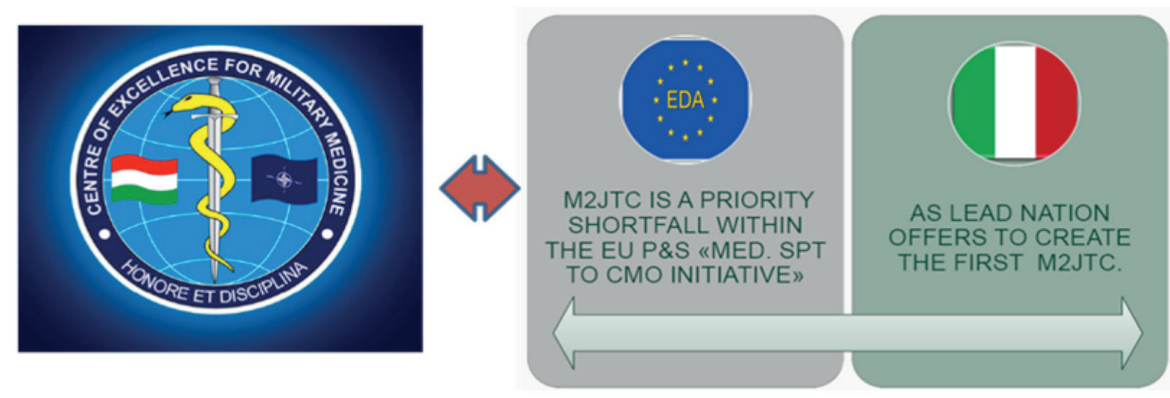

Figura 12. Relación Centros de Adiestramiento sanitario EDA-OTAN. tos que la configuran en su totalidad, incluyendo la cualificación y el adiestramiento del personal (Fig 11).

Por el momento, la colaboración y sinergia de ambas Iniciativas (Smart Defence y Pooling and Sharing), se están traduciendo, en la UE, en la creación de M3U,s bajo los referentes de la doctrina sanitaria de la Alianza Atlántica, la experiencia y lecciones aprendidas en operaciones combinadas y/o multinacionales, junto con el asesoramiento, adiestramiento e instrucción del Centro de Excelencia para la Sanidad Militar de la OTAN, en Budapest.

No obstante, las carencias doctrinales que la UE tiene en este proyecto de P\&S, están siendo subsanadas inicialmente, por el P\&SAHWG, mediante la adopción de normativa OTAN como punto de partida en la elaboración de la doctrina y normativa propias.

En tanto que no se dispone de un Centro de Adiestramiento Sanitario Multinacional dependiente de la EDA, Italia ha puesto a disposición de este organismo su Centro de Formación Sanitario (Roma) para ser utilizado por los diferentes países miembros de la iniciativa Pooling\&Sharing, como en el Multinational Medical Joint Training Centre (M2JTC) para que, en coordinación con el Centre of Excellence for Military Medicine (OTAN), de Budapest, constituya el primer Centro de la futura red de Centros de Formación, Adiestramiento e Instrucción de la EDA de los profesionales de la sanidad militar internacional que vayan a realizar rotaciones en las FSTM,s (Fig 12). 


\section{E. Chereguini Pavón y R.Blasco Ferrándiz}

Desde el seno de la EDA, se anima a que otros países se incorporen a esta Red de Centros de Adiestramiento e Instrucción Sanitaria para que puedan servir como elemento de integración, coordinación o aglutinación de conocimientos y experiencias, así como también para establecer estándares, formulación de propuestas, formación de equipos especializados y/o de evaluadores que participen en la acreditación de las MMTF/M3U (Fig 13).

La incorporación del Hospital de Campaña (HOC), al conjunto de FST, s de las FAS, puede constituir un hecho clave para establecer una revisión estratégica y funcional de la Sanidad Militar Española para normalizarla en los tres Ejércitos, representando un antes y un después de la Sanidad Operacional; un punto de inflexión a partir del cual se hace realidad prestar apoyo sanitario de Tercer Escalón Táctico a unidades tipo División y/o Cuerpo de Ejército (25.000 personas).

En virtud de lo anteriormente expuesto, podría ser un buen momento para realizar una reflexión de la Función Logística de la Sanidad Militar Española, en operaciones, desde un prisma integral, es decir, considerar los recursos y capacidades sanitarias de las FAS (ET, Armada y EA), como un todo normalizado, estructurado y protocolizado con un marcado carácter complementario e integrador que permita ser empleada indistinta $\mathrm{y} / \mathrm{o}$ conjuntamente por profesionales sanitarios del CMS, independientemente de su encuadramiento en uno o en otro Ejército.

Dado que la Sanidad Militar española viene participando con frecuencia y muy activamente en operaciones multinacionales de OI,s, en las que se aplican estándares y normativa sanitaria de la OTAN y en un futuro de la UE, parece oportuno que estos sean tenidos en cuenta e incorporados a la doctrina sanitaria de las FAS españolas, para su conocimiento y aplicación.

Igualmente, teniendo en cuenta los compromisos establecidos en sanidad operativa multinacional (proyectos Smart Defence (OTAN) y Pooling and Sharing (UE), sería conveniente considerar en próximos planeamientos sanitarios aquellas especificaciones técnicas, funcionales y de adaptación-acomodación, necesarias para hacer posible combinar y compartir los diferentes medios/equipos, de diferentes países, que van a constituir las futuras Formaciones Sanitarias de Tratamiento Multinacionales (MMTF/M3U).

En resumen, la Sanidad Militar Operacional en el contexto Multinacional está orientándose a un sistema funcional basado en el reparto de los esfuerzos para desarrollar el apoyo sanitario, en las operaciones conjuntas, apoyándose en el principio de "Reunir y Compartir" (Pooling and Sharing) medios, personal y/o capacidades sanitarias, para salvar el grave inconveniente que

le supone a un país realizar el esfuerzo del apoyo sanitario en solitario.

Las FSTM,s deben ir evolucionando en su concepto y capacidad, dado que en la actualidad las operaciones militares multinacionales convencionales se han reducido considerablemente y han surgido otras de naturaleza y objetivos diferentes que obligan a plantearse un nuevo concepto y planeamiento de las mismas, en función de lo "estrictamente necesario y suficiente", con una mayor cualificación y un reparto logístico y económico entre los países actuantes, según su grado de participación, sin olvidar que el objetivo principal de la Sanidad Militar, tanto nacional como multinacional, es prestar el apoyo sanitario al personal militar y/o civil autorizado, lo más cercano al punto del incidente, con la mayor rapidez y los mejores medios (humanos y materiales).

En definitiva, ofrecer en el TO una asistencia sanitaria de calidad equiparable a la que se desarrolla en territorio nacional.

\section{BIBLIOGRAFÍA}

1. Instrucción técnica $2 / 2013$ de 15 de Enero de la subsecretaría de defensa, por la que se asignan funciones y cometidos en el ámbito de la IGESANDEF y se describe su estructura orgánica, BOD nº 18/2013.

2. PD4-6161. "Sanidad en operaciones". Mando de Adiestramiento y Doctrina, Ejército de Tierra. Entrada en vigor 15-04-2011.

3. STANAG 2228 AJP-4.10 (A) "Joint Medical Support Doctrine".

4. STANAG 2552 MEDSTD ED1 RD1. Allied Medical Publication 56 (AMedP). "Guidelines for a Multinational Medical Unit. "NATO.

5. STANAG 2560 ED1 AMedP-27 "Manual de Evaluación Sanitaria” NATO.

6. Vázquez Ramos José A. "Mutualizar Capacidades Militares: Smart Defence y Pooling and Sharing." Revista Ejercito, 2013867 (7): 90-96.

7. Camille Grand, "Pooling and Sharing: conditions for long-term success". European Defence Matters. Issue 2, 2012.

8. Reinhard Marak. Senior Officer for Defence, Market, EDA. "Effective procurement can slash equipment costs". European Defence Matters. Issue 1, 2012 . 